\title{
Cavernous Body Reduction in Four Patients with Erectile Dysfunction Due To Insufficient Venous Occlusion and a Deficit of Elastic Fibers in the Tunica Albuginea
}

\author{
Fabrizio Iacono, Domenico Prezioso, Stefania Chierchia, Raffaele Galasso, Gennaro Iapicca, \\ Mario Di Martino
}

Section of Urology, School of Medicine, University of Naples “Federico II”, Naples, Italy

\begin{abstract}
Introduction: The corpora cavernosa are cylindrical vessels containing fluid under pressure. Thus, if cavernous wall resistance decreases, the radius increases and internal pressure decreases (LaPlace's law). We reasoned that if we decrease the corpus cavernosum radius, by excising a strip from each tunica albuginea, intracavernous pressure would increase during erection.

Materials and Methods: We treated with this procedure, four patients (mean age 41.5) with long-standing erectile dysfunction due to veno-occlusive dysfunction, non-responders to phosphodiesterase-5 inhibitors and intracavernous PGE1 injection.

Results: Two months post-surgery, intracavernous PGE1 (40 mcg) induced a satisfactory erection in two patients and a $45 \%$ and 58\% tumescence in the other two. PGE1 responders also responded to $100 \mathrm{mg}$ sildenafil. After $100 \mathrm{mg}$ sildenafil and 20 mg tadalafil, the two non-responders had erections that enabled penetration but were short lasting.

Conclusion: The procedure described could be more effective than cavernous revascularization operations. The results seem to confirm the mathematical assumptions.
\end{abstract}

Key words: erectile dysfunction; elastic fibers; tunica albuginea; corpora cavernosa

Int Braz J Urol. 2007; 33: 785-94

\section{INTRODUCTION}

Conservative surgical treatment (non-prosthetic) of erectile dysfunction (ED) targets the factors that most likely cause erectile failure and aims at restoring physiologic conditions. Unfortunately, this goal has only partly been achieved. For instance, the longterm outcome of corpus cavernosum microsurgical revascularization (1), which is aimed at augmenting endocavernous pressure during tumescence and penile rigidity, has been disappointing (2). Venous sur- gery has also proven to fail in the majority of cases (3).

Because the in- (arterial) and out- (venous) flow of blood within the corpus cavernosum is regulated by the trabecular smooth muscles and the tunica albuginea (TA) elastic properties, revascularization surgery will be unsuccessful if these components are damaged (4).

The severe reduction in elastic fibers in the TA of ED subjects seems to affect TA function $(5,6)$. Reduced TA elasticity could reduce TA resistance 
when intracavernous pressure as high as during erection (7). In fact, the corpora cavernosa are cylindrical vessels that contain fluid under pressure. According to Laplace's law, the larger the vessel radius the larger the wall tension required to withstand a given internal fluid pressure. Hence, an increase in the corpus cavernosum radius, consequent to reduced TA elasticity, would result in a decrease in internal pressure. Decreased TA elasticity may also reduce venous compression so leading to veno-occlusive dysfunction (VOD) and ED. Shafik et al. proposed a surgical technique involving the overlapping of the tunica albuginea to reduce the volume of the cavernous cylinder with encouraging results (8).

Here we describe a different surgical technique for $\mathrm{ED}$ in patients with VOD due to a reduction in the elastic fibers of the TA.

\section{MATERIALSAND METHODS}

For this treatment we selected four patients (mean age 41.5; range 24-57 y) who had suffered from severe ED due to veno-occlusive dysfunction (VOD) for more than 1 year, did not respond to the highest doses of phosphodiesterase-5 inhibitors nor to intracavernous PGE1 $20 \mathrm{mcg}$, and refused penile prosthetic implantation. All patients underwent medical history, IIEF, physical examination, blood serum tests, nocturnal penile tumescence (NPT) test for three consecutive nights, penile PGE1 Doppler flowmetry, pharmacocavernosometry. NPT test was considered normal when at least one erection event, lasting longer than 10 min and with a rigidity of more than $70 \%$ at the base and at the tip, was recorded $(9,10)$. Cavernous artery Doppler flowmetry was carried out in basal condition and after intracavernous stimulation with PGE1 (10 mcg) (11). Pharmacocavernosometry was performed according to Goldstein and Padma-Nathan (12).

Patients included were not smokers, nor affected by diabetes or other endocrine diseases.

In all patients, the diagnosis of VOD was confirmed by abnormal NPT test (Table-1), normal arterial function at Doppler flowmetry (Table-2), dysfunctional pharmacavernosometry with absence of erectile response at increasing intracavernous doses of PGE1 up to $60 \mathrm{mcg}$.

After rejecting penile implantation, patients were proposed cavernous body reduction technique as a "last resource" procedure. They were asked to sign an informed consent form and the authorization of the Hospital Ethics Committee was obtained.

Before undergoing surgery, all patients underwent a biopsy of the tunica albuginea with the biopsygun technique $(6,13,14)$. The TA specimens were fixed in $10 \%$ buffered formalin solution and embedded in paraffin, and a $5-\mu$ section was stained with hematoxylin and eosin, and Weigert's stain (for elastic fibers). Elastic fibers were counted on 10 to 12 fields (40 X magnification) on five serial sections (10 to 12 fields for each section). Routine counting techniques were used, namely, mitotic counting, or more specifically, AgNOR counting (15). All patients showed a severe reduction of the TA elastic fibers, i.e., between 27.77 and 49.32 for each high power field $(6,13)$.

Table 1 - Results of RigiScan nocturnal tumescence monitoring. The erectile episodes rate during 3 nights of registration (frequency), the duration of the best erectile episode (duration/min.) and the base and tip rigidity percentage of the best erectile episode, before (pre) and after (post) surgery.

\begin{tabular}{llccccccccc}
\hline & Age & \multicolumn{2}{c}{ Frequency } & \multicolumn{2}{c}{ Duration/min } & \multicolumn{2}{c}{ Rigidity } \\
& & Pre & Post & Pre & Post & & Tip & & Base \\
& & & & & & Pre & Post & Pre & Post \\
\hline Pte. 1 & 24 & 1 & 2 & 3 & 12 & 52.3 & 68.8 & 59.1 & 70.2 \\
Pte. 2 & 39 & 2 & 3 & 6 & 10 & 60.4 & 65.6 & 65.7 & 73.4 & \\
Pte. 3 & 46 & 4 & 3 & 2 & 11 & 35.7 & 58.1 & 39.0 & 60.8 \\
Pte. 4 & 57 & 1 & 3 & 5 & 14 & 55.3 & 71.0 & 58.4 & 70.1 \\
\hline
\end{tabular}


Table 2 - Echo Doppler flowmetry evaluation.

\begin{tabular}{|c|c|c|c|c|c|}
\hline & \multicolumn{2}{|c|}{ Arterial Diameter/mm } & \multicolumn{3}{|c|}{ Flow Rate, $\mathrm{cm} / \mathrm{sec}$} \\
\hline & Before Injection & After Injection & Peak & Diastolic & Resistance Index \\
\hline \multicolumn{6}{|l|}{ Pte. 1} \\
\hline Right art. & 0.43 & 0.85 & 45.33 & 13.44 & 0.70 \\
\hline Left art. & 0.43 & 0.85 & 44.66 & 13.30 & 0.70 \\
\hline \multicolumn{6}{|l|}{ Pte. 2} \\
\hline Right art. & 0.39 & 0.80 & 41.23 & 11.55 & 0.68 \\
\hline Left art. & 0.40 & 0.81 & 41.00 & 11.40 & 0.67 \\
\hline \multicolumn{6}{|l|}{ Pte. 3} \\
\hline Right art. & 0.41 & 0.79 & 45.45 & 12.34 & 0.71 \\
\hline Left art. & 0.41 & 0.79 & 45.12 & 12.50 & 0.65 \\
\hline \multicolumn{6}{|l|}{ Pte. 4} \\
\hline Right art. & 0.45 & 0.94 & 45.00 & 13.50 & 0.77 \\
\hline Left art. & 0.45 & 0.94 & 45.00 & 13.51 & 0.77 \\
\hline
\end{tabular}

Eight to 10 weeks after surgery, the four patients underwent a NPT test and a PGE1 intracavernous test with real-time rigidometry, and then were allowed to assume PDE5 inhibitors and attempt a sexual intercourse. Follow-up visits took place 3 and 6 months after surgery.

\section{Surgical Procedure}

Under general anesthesia, a subcoronal incision is performed and the penis is skinned to the base. Buck's fascia is longitudinally opened with scissors along both lateral faces of the shaft, from penis basis to balanopreputial sulcus, approximately $1 \mathrm{~cm}$ dorsally to the spongy body of the urethra. The cleavage plane beneath the fascia is bilaterally developed, circumflexes veins are ligated and the surface of the tunica albuginea is exposed. A haemostatic tourniquet is placed at the penis base and erection is induced through intracavernous saline infusion. The strip to be removed from the TA, 0.5 to $1 \mathrm{~cm}$ wide and just as long as the shaft is, are then marked on both faces by a demographic pencil. The tourniquet is removed consenting detumescence and then placed again to reduce bleeding. The TA is incised with a \#11 blade along the marked traces (Figure-1). The strips are then detached from the underlying cavernous tissue with blunt-tipped scissors (Figure-2) and removed (Figure-3). The TA margins are sutured by a PDS 4-0 running suture (Figure-4). Buck's fascia is closed through a biosyn 3-0 interrupted suture. Dartos fascia and skin are reconstructed with biosyn 4-0 interrupted sutures.

An 18F Foley catheter is left in place for 24 hours and a compressive bandage is applied for two days. During surgery a single bolus of antibiotics, $2 \mathrm{~g}$ ceftazidime, is administered. To inhibit nocturnal erections, a $20 \mathrm{mg}$ bedtime dose of diazepam is administered in the first 7 days and an intramuscular $100 \mathrm{mg}$ dose of cyproterone acetate is administered once a week for three weeks.

\section{RESULTS}

The postoperative course was uneventful in all patients. Two patients reported some pain during moderate spontaneous tumescence while sleeping during the first 20 days after surgery. The pain disappeared spontaneously.

All patients reported a relevant spontaneous penile tumescence while sleeping or upon awaking 


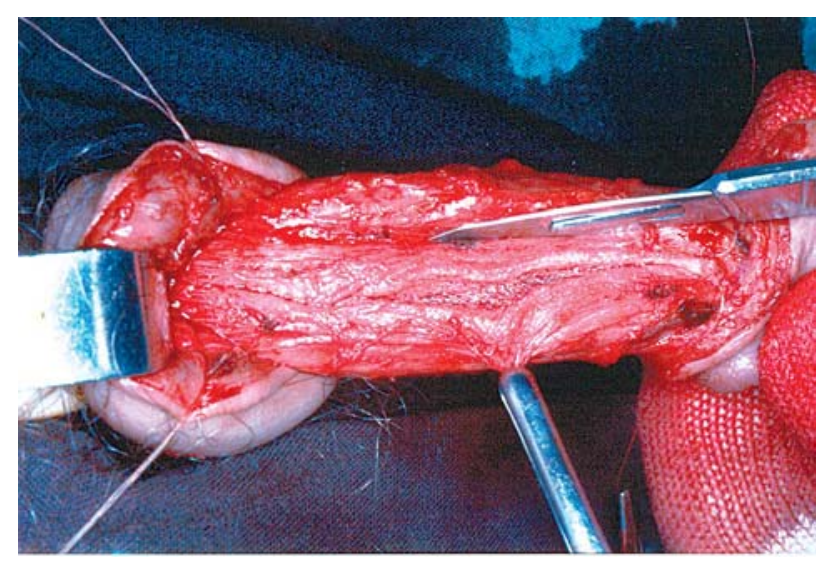

Figure 1 - Incision of the tunica albuginea on the left corpus cavernosum. A longitudinal band in the tunica (of about $0.5 \mathrm{~cm}$ ) is prepared.

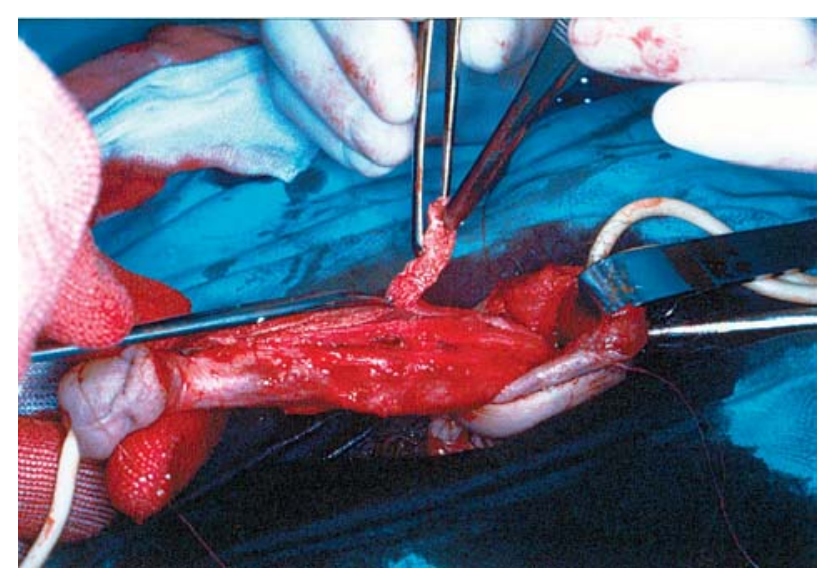

Figure 2 - The band in the tunica albuginea is excised. The erectile tissue is separated from the tunica with the tip of a scissors.

four weeks after surgery. Eight to ten weeks after surgery the NPT test showed a clear improvement in all patients (Table-1) and the PGE1 (40 mcg) test with real-time rigidometry showed: in two patients, a satisfactory erection both in rigidity (> 70\%) and duration (>10 $\mathrm{min}$ ); in the other two patients, a rigidity of $45 \%$ and 58\% lasting 15-20 min.

At the 3 months follow-up visits the two PGE1 responders reported good erection with sildenafil citrate $100 \mathrm{mg}$ or tadalafil $20 \mathrm{mg}$, subjectively perceived as satisfactory in terms of both rigidity and duration. The two low-responders to intracavernous PGE1 injection had partial response to $100 \mathrm{mg}$ sildenafil or 20

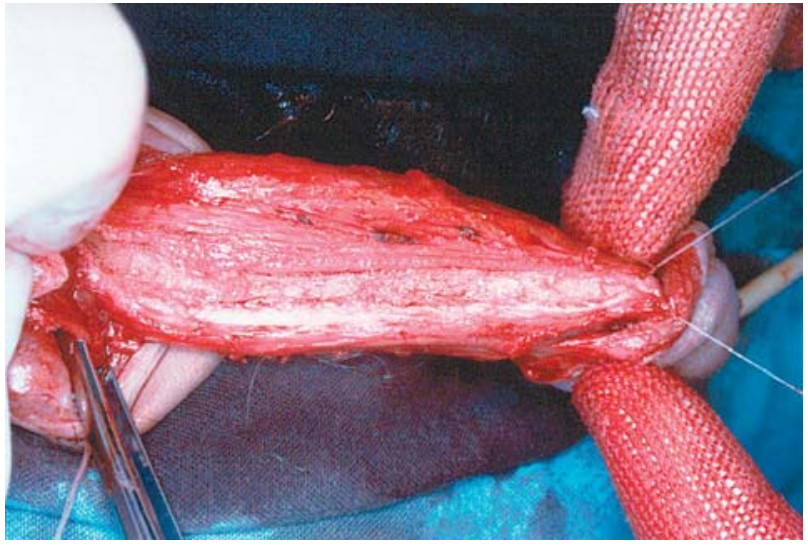

Figure 3 - Ventral face of the left corpus cavernosum. A band in the tunica albuginea has been removed.

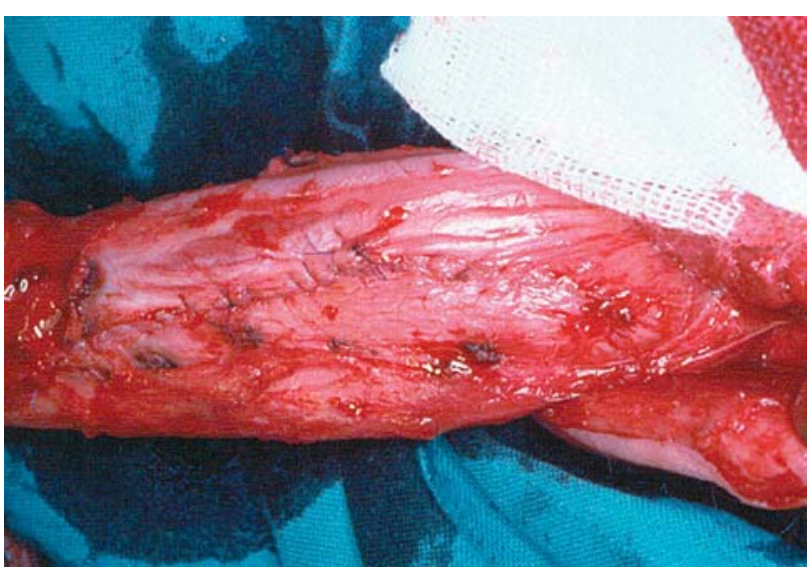

Figure 4- Continuous suture by PDS 4-0 of the tunica albuginea on the ventral face of the left corpus cavernosum.

mg tadalafil, reporting partial erection barely sufficient for vaginal penetration and of insufficient duration for a satisfactory sexual intercourse.

Overall, all patients were satisfied with the surgery. None reported functional or psychological consequences due to the reduced penile cross-section. All declared that they did not feel any difference and that they would undergo the operation again.

\section{COMMENTS}

During penile erection, tumescence is caused by smooth muscle relaxation, which exposes the lacu- 
nar spaces to systemic systolic arterial blood pressure thus inducing blood engorgement of corpora cavernosa. As penile volume maximizes secondary to cavernous tissue and, principally, tunica albuginea compliance, further increment in intracavernous blood pressure will translate into increased rigidity. Penile rigidity is then mainly influenced by intracavernous pressure (16). However it has been shown that a wide range of pressure can be associated with penile rigidity, and if most patients achieve full rigidity when intracavernous pressure approaches $60-90 \mathrm{mmHg}$, in some cased rigidity is observed at $40-50 \mathrm{mmHg}$ pressure, while in some other pressure exceeding $120 \mathrm{mmHg}$ are needed. Penile geometry is considered an important factor in explaining such variability, and it has been clearly suggested that both length and diameter influence the intracavernous pressure needed to achieve rigidity, and that at a given length, in presence of a bigger diameter a higher pressure is required $(17,18)$.

Our preliminary experience with this novel technique seems to support a rationale for a cavernous body reduction in patients with severe erectile dysfunction non responsive to oral or injectable ED drugs. All four patients improved their erectile function after the procedure, although only in two cases the response was considered satisfactory at the postoperative real-time rigidometry. From a clinical viewpoint, patients regained good or partial responsivity to PDE5 inhibitor that in two cases translated in satisfactory sexual activity.

All patients were affected with VOD, and presented good arterial function. We think these patients may prove to be the best candidate for this procedure, although some efficacy in cases of arteriogenic dysfunction might be shown.

Cavernous body reduction is a simple and quick procedure, with minimal side effects. Nevertheless, it can be proposed only to a very limited subgroup of patients, i.e. patients with severe ED non responsive to pharmacological treatment, where a good arterial function can be documented, who refuse penile prosthesis but not "conservative" procedures that could possibly improve the drug-assisted erection. Patients have also to accept a permanent reduction of penis girth, although in our experience this can be barely perceived after the procedure.

\section{CONCLUSION}

As penile geometry is an important factor in determining erectile function, a tunica albuginea reduction can improve erectile function in patients with severe ED from veno-occlusive dysfunction. This experimental procedure can be proposed as a "last resource" technique in patients affected with severe $\mathrm{ED}$, who are bothered enough to warrant a surgical approach but refuse penile implantation. Further experience is needed to better define short and longterm results of this procedure, and to identify the preoperative factors that can indicate or contraindicate its application.

\section{ACKNOWLEDGEMENT}

Jean Ann Gilder edited the text.

\section{CONFLICT OF INTEREST}

None declared.

\section{REFERENCES}

1. Michal V, Kramar R, Hejhel L: Revascularization procedures of the cavernous bodies. In: Proceedings of the First International Conference on Cavernous Bodies Revascularization. Charles C. Thomas, Springfield, Ill. 1980; pp. 239.

2. Wespes E, Wildschutz T, Roumeguere T, Schulman CC: The place of surgery for vascular impotence in the third millennium. J Urol. 2003; 170: 1284-6.

3. Popken G, Katzenwadel A, Wetterauer U: Long-term results of dorsal penile vein ligation for symptomatic treatment of erectile dysfunction. Andrologia. 1999; 31 (Suppl 1): 77-82.

4. Lewis RW: Arteriovenous Surgeries: Do They Make Any Sense? In: Lue TF, (ed.), World Book of Impotence. London, Smith Gordon, 1992; pp. 199.

5. Goldstein AM, Meehan JP, Morrow JW, Buckley PA, Rogers FA: The fibrous skeleton of the corpora cavernosa and its probable function in the mechanism of erection. Br J Urol. 1985; 57: 574-8. 
6. Iacono F, Barra S, de Rosa G, Boscaino A, Lotti T: Microstructural disorders of tunica albuginea in patients affected by impotence. Eur Urol. 1994; 26: 233-9.

7. Bitsch M, Kromann-Andersen B, Schou J, Sjontoft E: The elasticity and the tensile strength of tunica albuginea of the corpora cavernosa. J Urol. 1990; 143: 642-5.

8. Shafik A, Shafik I, El Sibai O, Shafik AA: Tunica albuginea overlapping: a novel technique for the treatment of erectile dysfunction. Andrologia. 2005; 37: 180-4.

9. Kessler WO: Nocturnal penile tumescence. Urol Clin North Am. 1988; 15: 81-6.

10. Seoung IG, Choi HK. Reliability of Rigiscan for evaluating erectile failure. Int J Impotence Res 1990; (suppl 1): pp 191-197.

11. Barra S, Iacono F: Echo-Doppler-flowmetric assessment of penile dorsal arteries and their role in the erectile mechanism. Eur J Radiol. 1997; 25: 67-73.

12. Goldstein I, Padma-Nathan H: The interaction of arterial and venous hemodynamics during erection: dynamic infusion cavernosometry and cavernosography. AUA, New England Sect 1987; pp. 18 (abstract).
13. Iacono F, Barra S, De Rosa G, Boscaino A, Lotti T: Microstructural disorders of tunica albuginea in patients affected by Peyronie's disease with or without erection dysfunction. J Urol. 1993; 150: 1806-9.

14. Wespes E, Depierreux M, Schulman CC: Use of bBiopty gun for corpus cavernosum biopsies. Eur Urol. 1990; 18: 81-3.

15. De Rosa G, Staibano S, Barra E, Zeppa P, Salvatore G, Vetrani A, et al.: Nucleolar organizer regions in aggressive and nonaggressive basal cell carcinoma of the skin. Cancer. 1992; 69: 123-6.

16. Lavoisier P, Proulx J, Courtois F: Reflex contractions of the ischiocavernosus muscles following electrical and pressure stimulations. J Urol. 1988; 139: 396-9.

17. Desai KM, Floyd TJ, Follett DH, Peake DR, Gingell JC: Development of a penile rigidity indicator and new concepts in the quantification of rigidity. $\mathrm{Br} \mathrm{J}$ Urol. 1988; 61:254-60.

18. Puech-Leao P, Akira S, Chao S: Penile architecture and intracavernous pressure: a simulation. Int J Impot Res. 1997; 4 (suppl 2), 43.
Accepted after revision:

June 26, 2007

\author{
Correspondence address: \\ Dr. Fabrizio Iacono \\ Via Tasso 480, 80127 \\ Naples, Italy \\ Fax: + 3908 1060-9149 \\ E-mail: fiacon@tin.it
}

\section{EDITORIAL COMMENT}

The authors report an interesting experience with a new surgical technique (cavernous body reduction) in 4 patients suffering from severe erectile dysfunction (ED) non-responsive to oral or injectable ED drugs. In all the patients the diagnosis of venoocclusive dysfunction (VOD) was confirmed by ab- normal nocturnal penile tumescence (NPT) test, dynamic Doppler flowmetry, blood examination and pharmaco-cavernosometry.

Before undergoing surgery, all patients underwent a biopsy of tunica albuginea (TA) with the biopsy-gun technique; the TA specimens showed a severe reduction of TA elastic system fibers. 
The technique described by the authors is inspired by Shafik's work. Shafik et al. proposed a surgical technique involving the overlapping of TA to reduce the cavernous cylinder volume with encouraging results. The aim of this work is to highlight the role of TA in the erectile mechanism; in fact, we agree that TA is not a pure holder of corpora cavernosa, but its integrity is crucial for the physiology of erection.

We just propose some considerations about patients' inclusion criteria. The diagnosis of veno-occlusive dysfunction in patients affected by ED without any other possible cause is moreover a big challenge for andrologists:

- The selected patients have suffered from ED for more than 1 year, but it is not clear if the sexual dysfunction is an acquired or a congenital disease; in fact, the onset of severe acquired ED due to reduction of elastic fibers could be related to other systemic or metabolic affections. Have endocrine blood examinations been performed? As well as an important step in the management of andrological patient, an androgen deficiency, also partial, could be responsible for a diminished trophism in many organs, including TA.

- The relationship between reduction of TA elasticity, TA resistance and the corpus cavernosum radius it is not clear. TA elasticity, as we believe, is a crucial point to induce detumescence with a ten fold increase of its thickness.

- The Authors do not report any consideration about psychological assessment concerning sexological aspects. We believe that this evaluation has a precise role in order to identify patients with high lev-

\section{EDITORIAL COMMENT}

The article "Cavernous Body Reduction in Four Patients with Erectile Dysfunction Due To Insufficient Venous Occlusion and a Deficit of Elastic els of anxiety or depression. In fact it is well known that these conditions determines a series of biochemical changes in brain and body; particularly sympathetic hyperactivity with increased blood levels of catecholamines induces vasoconstriction and increased penile smooth muscle tone. In these patients pharmaco-cavernosometry can give false results. Moreover, these patients are frequently affected by sleep disorders. It would have been interesting to perform a nocturnal polysomnogram test that, together with nocturnal penile tumescence test, could lead to a more certain diagnosis.

The surgical technique is mainly based on a volumetric reduction of corpora cavernosa and implies an irreversible alteration of penile anatomy.

Our major objections to the authors are the following:

- Follow up is too short to obtain certain results (moreover they are not successful as reported by the authors themselves); in fact a partial and brief success of the technique could be related to ligation of circumflexes veins (as reported in literature).

- At that moment the patients did not accept penile prosthesis implant; but were they informed that cavernous body reduction could compromise, in the future, this kind of surgery?

In conclusion, this article is really interesting for an innovative approach to ED patients non-responders to oral or injectable drugs. But a short follow up may have biased the findings of the authors. It would be interesting to be informed about the future clinical condition of these patients.

\section{Dr. Marco Grasso \\ Department of Urology, Desio Hospital San Raffaele Hospital, Milan \\ E-mail:marco.grasso@aovimercate.org}

Fibers in the Tunica Albuginea" is very similar to the published article "Shafik A, Shafik I, El Sibai O, Shafik AA: Tunica albuginea overlapping: a novel technique 
for the treatment of erectile dysfunction. Andrologia. 2005; 37: 180-184". This technique in our study was applied on nine patients instead of four patients in this study; we assumed that authors would increase the number of patients in order to provide the readers with better statistical analysis or to prolong the follow up period for more evaluation of the outcome. The title is deceiving, in which it appears as a new technique. The aim of work is not clear weather to our technique [according to Shafik technique] or to compare with other techniques. The discussion is poor in comparison to our article on 2005. In fact, our novel technique is based on histopathologic study demonstrating the pathogenesis of tunica albuginea in venogenic erectile dysfunction patients, in which the technique is dealing with the pathology. Since our aforementioned article we reached 24 patients underwent that technique and we are about to publish the results.

\section{Dr. Ali A. Shafik \\ Associate Professor of Surgery \\ Cairo University \\ Cairo, Egypt \\ E-mail:shafik@ahmedshafik.com}

\section{REPLY BY THE AUTHORS}

We presented our surgical technique for the first time at the National Congress of the Italian Society of Urologists in June 2004 and reported the initial data (1). The Shafik et al. publication came out a year later. Shafik's surgical technique, although part of a similar rationale and one, therefore, that supports our theories which have already been published on several occasions, involves overlapping of the albuginea rather than the removal of a piece of tissue. Overlapping, in our opinion, leads to thickening of the corpus cavernosum and reduced longitudinal expansion of the penis during erection.

The four patients operated on all suffered from erectile deficit of organic origin as was revealed by the diagnostic tests carried out and by the lack of response to the vasoactive drugs administered. No psychological assessment would have altered the diagnosis and so it was not considered appropriate to carry out psychological tests. There may have been a psychological component overlaying the obvious organic etiology but this would not have affected the therapy in any way. The endocrine blood examination of all patients was performed. No endocrine or metabolic disease was identified.
We were the first to demonstrate (2-5) and then other authors did the same $(6,7)$ that patients presenting with erectile dysfunction (ED) are affected by a damaged tunica albuginea (TA) with reduced elastic fibers. Numerous authors have demonstrated the function of the elastic fibers in the microstructure of the tunica albuginea. It appears that a reduction in the elastic fibers in the tunica albuginea of the corpus cavernosum affects the veno-occlusive function of the tunica. Why patients with ED have reduced elastic fibers is not yet known. It may be that it is an acquired syndrome, but we do not know.

Our own experience, based on dozens of surgical operations carried out by our team to try and solve cases of veno-occlusive dysfunction ED, involving massive ligature of the venous cavernous area, including stripping of the penal dorsal vein, and ligature of the crural and all circumflex veins, has shown that results are very disappointing even one month post surgery. What is more, results published by numerous other authors demonstrate the failure of these surgical procedures both in the short as well as in the long term (8-11). It is our opinion that simple ligature of a vein in the corpus 
cavernosum cannot (unfortunately) improve erectile function in any way.

Normally the arterial blood flow reaching the two corpora cavernosa through the right and left cavernous arteries corresponds to the amount of venous blood flowing back from the cavernous circulation leaving the penis through the triple venous system.

One of the main vascular mechanisms, which can determine the pressure increase inside the penis and, lastly, the erection, is the trans-albugineal veins compression. These veins are compressed by the TA while the corpora cavernosa fill in, resulting in a strong reduction of the blood drainage from the corpora cavernosa. The penis fills in as a consequence of the flow rate increase, since the excitement phase leads to an increase in both the vessel diameter and the systolic rate.

The flow rate is expressed by the following formula $\mathrm{Q}=\mathrm{A}^{*} \mathrm{v}$ [1] where ' $\mathrm{A}$ ' is the vessel crosssection and ' $\mathrm{v}$ ' is the mean rate. Since $\mathrm{v}=\mathrm{DP} * \mathrm{r} 2 / 8 \mathrm{nl}$ [2] where ' $n$ ' is the blood viscosity, the replacement of [1] with [2] yields Q = DP 3.14 r4/8nl (2 a) which clearly helps to understand that even a slight vessel ray increase could dramatically augment the flow rate.

The progressive pressure increase inside the corpora cavernosa compresses the trans-TA venules, reducing the flow rate and generating an imbalance between the Qi (in- flow rate) and the Qu (out- flow rate). Therefore Qi > Qu.

Under normal conditions La Place's law explains the tension generated on the corpora cavernosa wall structure, i.e. on the TA, assuming that the two corpora cavernosa can be considered as infinitely long cylinders, so $\mathrm{r} 2=\infty$, also deducing that $\mathrm{p}=\mathrm{t} / \mathrm{r} 1$ or also that $\mathrm{t}=\mathrm{p} * \mathrm{r} 1$.

There is an important consequence of this law: in order to reach an established pressure inside a container, a higher tension is required if the curvature ray is higher.

It is then clear enough how fundamental the role played by the TA is in the erectile phenomenon. The baseline TA structure is made up of wavy looping collagen bands, and of elastic fibers bridging the loops while providing elasticity to the collagen structure. During the erectile event, the loops would be changed only slightly in their fundamental structure.
Nonetheless, there is still an increase in the corpus cavernosum diameter, which is regulated by Young's module, where we notice: $\mathrm{DR}=\mathrm{P} * \mathrm{r} 2 / 2 \mathrm{E}^{*} \mathrm{~s} *(2-\mathrm{v})$ [3] where ' $E$ ' is the elasticity coefficient and ' $v$ ' is Poisson's coefficient.

Thanks to this law, it is clear that, if pressure remains stable, a cylinder wall elasticity decrease corresponds to a cylinder curvature ray increase. By this formula, $\mathrm{P}$ can be obtained, $\mathrm{P}=2 \mathrm{E}^{*} \mathrm{~s} * \mathrm{Dr} / \mathrm{r} 2 *$ (2v) [4]. It is remarkable that a minimal cylinder ray increase results in a substantial reduction of the inner cylinder pressure. It is obvious that the higher the study material elasticity, the higher the structure inner pressure. A reduction of the elastic fibers inside the TA would cause a change in the normal collagen loops structure: their flattening would result in a longer period, an overall increase in the cylinder structure ray and a subsequent pressure drop by stable flow (erectile failure).

The authors do not believe that the cavernous body reduction could compromise future penile prosthesis implant.

\section{REFERENCES}

1. Iacono F, Barra S, Giannella R, Manno G, Di Lauro G: Riduzione volumetrica chirurgica dei corpi cavernosi nell'insufficienza veno-occlusiva e deficit di fibre elastiche nella tunica albuginea. Atti $77^{\circ}$ Congresso Nazionale della Società Italiana di Urologia. Abst. P27, pp. 92, 2004.

2. Iacono F, Barra S, De Rosa G, Boscaino A, Lotti T: Microstructural disorders of tunica albuginea in patients affected by Peyronie's disease with or without erection dysfunction. J Urol. 1993; 150: 1806-9.

3. Iacono F, Barra S, De Rosa G, Boscaino A, Lotti T: Microstructural disorders of tunica albuginea in patients affected by impotence". Eur Urol. 1994; 26: 233-9.

4. Iacono F, Barra S, Lotti T: Elastic fibers concentration in tunica albuginea of corpora cavernosa and nocturnal tumescence monitoring. Int J Imp Res. 1995; 7: 63-70.

5. Iacono F, Barra S, Cafiero G, Lotti T: Scanning electron microscopy study of tunica albuginea of corpora cavernosa in normal and impotent subjects. Urol Res. 1995; 23: 221-6.

6. Sattar AA, Wespes E, Schulman CC: Computerized measurement of penile elastic fibres in potent and impotent men. Eur Urol. 1994; 25: 142-4. 
7. Akkus, E, Carrier S, Baba K, Hsu GL, Padma-Nathan H, Nunes L, Lue TF: Structural alterations in the tunica albuginea of the penis: impact of Peyronie's disease, ageing and impotence. Br J Urol. 1997; 80: 190.

8. PopkenG, Katzenwadel A, Wetterauer U: Long term results of dorsal vein ligation for symptomatic treatment of erectile dysfunction. Andrologia. 1999; 31 (suppl. 1): 77-82.

9. Gilbert P, Sparwasser C, Beckert R, Treiber U, Pust R: Venous surgery in erectile dysfunction. The role of dorsal penile vein ligation and spongiosolysis for impotence. Urol Int.1992; 49: 40-7.

10. Sun XJ: Diagnosis and surgical tretment of impotence caused by venous leakage. Zhonghua Wai Ke Za Zhi [Chinese journal of surgery]. 1990; 28: 554-7.

11. Freedman AL, Costa Neto F, Mehringer CM, Rajfer J: Long term results of penile vein ligation for impotence from venous leakage. J. Urol. 1993; 149:1301-3. 